\title{
Evaluation of biological pathways involved in chemotherapy response in breast cancer
}

\author{
Attila Tordai ${ }^{1,4}$, Jing Wang ${ }^{2}$, Fabrice Andre ${ }^{5}$, Cornelia Liedtke ${ }^{1}$, Kai Yan ${ }^{2}$, Christos Sotiriou ${ }^{6}$, Gabriel N Hortobagyi ${ }^{1}$, \\ W Fraser Symmans ${ }^{3}$, Lajos Pusztai ${ }^{*}$
}

\begin{abstract}
Introduction: Our goal was to examine the association between biological pathways and response to chemotherapy in estrogen receptor (ER)-positive and ER-negative breast cancers separately.

Methods: Gene set enrichment analysis including 852 predefined gene sets, was applied to gene expression data from 51 ER-negative and 82 ER-positive breast cancers that were all treated with a preoperative paclitaxel, 5-fluoruracil, doxorubicin and cyclophosphamide chemotherapy.

Results: Twenty-seven (53\%) ER-negative and 7 (9\%) ER-positive patients had pathologic complete response (pCR) to therapy. Among the ER-negative cancers, a proliferation gene signature (FDR $q=0.1$ ), the Genomic Grade Index $(F D R q=0.044)$ and the E2F3 pathway (FDR $q=0.22, p=0.07)$ signature were enriched in the $p C R$ group. Among the ER-positive cancers, the proliferation signature (FDR $q=0.001)$ and the Genomic Grade Index (FDR $q=0.015$ ) were also significantly enriched in cases with pCR. Ki67 expression, as single gene marker of proliferation, did not provide the same information as the entire proliferation signature. An ER-associated gene set (FDR $q=0.03$ ) and a mutant p53-gene signature (FDR q $=0.0019)$ were enriched in ER-positive cancers with residual cancer.

Conclusion: Proliferation- and genomic grade-related gene signatures are associated with chemotherapy sensitivity in both ER-negative and -positive breast cancers. Genes involved in the E2F3 pathway are associated with chemotherapy sensitivity among ER-negative cancers. The mutant p53-signature and expression of ER-related genes were associated with lower sensitivity to chemotherapy in ER-positive breast cancers only.
\end{abstract}

\section{Introduction}

Drug resistance is caused by multiple mechanisms that operate simultaneously in cancers. A large number of biological functions including transmembrane trafficking, DNA-repair, stress response, proliferation and apoptosis may affect the sensitivity of a cell to chemotherapy. Other, yet to be identified mechanisms may also play a role. Preoperative chemotherapy provides an attractive clinical setting to study mechanisms of drug resistance in patients.

Chemotherapy before surgery is used in the treatment of newly diagnosed, stage II-III breast cancers because it frequently reduces tumor size and improves surgical outcome [1]. Among patients who receive preoperative chemotherapy, up to $25-30 \%$ (depending on the type of

\footnotetext{
* Correspondence: Ipusztai@mdanderson.org

'Department of Breast Medical Oncology, The University of Texas M. D.

Anderson Cancer Center, Houston, TX

Full list of author information is available at the end of the article
}

treatment) experience complete eradication of the invasive cancer in the breast and regional lymph nodes after completion of 3-6 months of chemotherapy [2]. This favorable response is called pathologic complete response $(\mathrm{pCR})$ and it indicates an extremely chemotherapy sensitive tumor and also heralds excellent long-term cancer-free survival [3]. We previously conducted a pharmacogenomic study that included 133 patients with newly diagnosed breast cancer who received preoperative chemotherapy with paclitaxel followed by 5 -fluorouracil, doxorubicin and cyclophosphamide. All patients underwent a one-time, pre-treatment fine needle biopsy of the cancer for gene expression analysis. The goal of the study was to discover geneexpression based predictors of pCR. Our previous analysis focused on discovering the best possible multi-gene predictor without considering the function of any of the genes [4]. The goal of the current analysis is to examine 
an association between known biological pathways and response to chemotherapy.

Lists of genes (i.e. gene sets) that represent various biological pathways were assembled from the literature. We used gene set enrichment analysis (GSEA) to examine the correlation between these a priori defined gene sets and chemotherapy response [5]. Clinical experience as well as molecular analysis of breast cancers indicate that estrogen receptor (ER)-positive and ER-negative cancers are two different types of neoplastic diseases of the breast therefore [6,7]. It is plausible that different molecular mechanisms may determine response or resistance to chemotherapy in these 2 types of breast cancers. Therefore, we performed our analysis separately for ER-positive and ER-negative cancers.

\section{Materials and methods Patients}

This study included 51 ER-negative and 82 ER-positive tumors from patients with newly diagnosed stage I-III breast cancer. Each patient had a fine needle aspiration of the cancer before starting chemotherapy. These needle aspiration samples contain approximately $80 \%$ neoplastic cells and few or no stromal cells or normal breast epithelium [8]. All patients were treated with 6 months of preoperative chemotherapy with paclitaxel followed by 5 fluorouracil, doxorubicin and cyclophosphamide. Patients underwent surgery after completion of chemotherapy and the resection specimens were examined by a pathologist to measure residual cancer. For the purpose of our analysis, tumor response was dichotomized as $\mathrm{PCR}$, defined as no residual invasive cancer in the breast and lymph nodes, or residual disease (RD) that included patients with any degree of invasive cancer that survived preoperative chemotherapy. The reason for this dichotomization was because $\mathrm{pCR}$ is a strong surrogate for long term cancerfree survival and therefore a marker of long-term benefit from therapy $[2,3]$. It remains unknown to what extent patients who achieve less than PCR benefit from chemotherapy in terms of improved survival. This categorization of pathologic response allowed us to compare biological pathways between cancers with extreme chemotherapy sensitivity (pCR) and the rest (RD). There were not enough cases with tumor progression during treatment in our study to form a third group including extreme chemotherapy resistant tumors.

ER-status was determined from routine pathological assessment by immunohistochemistry. The cut-off for ER-positivity was $\geq 10 \%$ positive tumor cells following standard clinical practice. This study was approved by the institutional review boards (IRB) of MDACC and all patients signed an informed consent for voluntary participation. Clinical characteristics of the patients are presented in Table 1.
Table 1 Patient characteristics

\begin{tabular}{|c|c|c|}
\hline Characteristics & $\begin{array}{c}\text { ER-negative } \\
\text { group } \\
(n=51)\end{array}$ & $\begin{array}{c}\text { ER-positive } \\
\text { group } \\
(n=82)\end{array}$ \\
\hline $\begin{array}{l}\text { Median Age (years) } \\
\text { Range }\end{array}$ & $\begin{array}{c}51 \\
(29-75)\end{array}$ & $\begin{array}{c}51 \\
(28-79)\end{array}$ \\
\hline \multicolumn{3}{|l|}{ T stage } \\
\hline TO & 0 & $1(1 \%)$ \\
\hline $\mathrm{T} 1$ & $8(16 \%)$ & $4(5 \%)$ \\
\hline $\mathrm{T} 2$ & $24(47 \%)$ & $46(56 \%)$ \\
\hline $\mathrm{T} 3 / 4$ & $19(37 \%)$ & $31(38 \%)$ \\
\hline \multicolumn{3}{|l|}{ Histological grade } \\
\hline Grade 1 & 0 & $2(2 \%)$ \\
\hline Grade 2 & $6(12 \%)$ & $45(55 \%)$ \\
\hline Grade 3 & $43(84 \%)$ & $31(38 \%)$ \\
\hline Unknown & $2(4 \%)$ & $4(5 \%)$ \\
\hline \multicolumn{3}{|l|}{ Lymph node status } \\
\hline Positive & $38(75 \%)$ & $55(67 \%)$ \\
\hline Negative & $13(25 \%)$ & $27(33 \%)$ \\
\hline \multicolumn{3}{|c|}{$\begin{array}{l}\text { HER2 over-expressed or } \\
\text { amplified }\end{array}$} \\
\hline Yes & $18(35 \%)$ & $15(18 \%)$ \\
\hline No & $32(63 \%)$ & $67(82 \%)$ \\
\hline Unknown & $1(2 \%)$ & 0 \\
\hline \multicolumn{3}{|c|}{ Pathologic complete response } \\
\hline Yes & $27(53 \%)$ & $7(9 \%)$ \\
\hline No & $24(47 \%)$ & 75 (91\%) \\
\hline
\end{tabular}

\section{Gene expression analysis}

Gene expression profiling was performed by using Affymetrix U133A Gene Chips following standard operating procedures as described previously [4]. We normalized the gene expression data using dChip V1.3 software [9] to a single reference array. The normalized gene expression values were transformed to a $\log _{10}$ scale for further analysis. The complete microarray data is available at the M.D. Anderson Cancer Center bioinformatics web site [10]. To identify differentially expressed genes between cases with pCR and $R D$ we performed unequal variance $t$-test on each probe set. Because of the multiple comparisons many low p-values are expected by chance alone. Under the null hypothesis that no genes provide useful information, the distribution of p-values should be uniform. If, on the other hand, some genes do provide useful information about predicting response, we would expect an overabundance of small p-values (above what chance might produce). We can capture this situation by modeling the distribution of the p-values as a beta-uniform mixture (BUM). This analysis was used to estimate false discovery rates (FDR) that accompany particular $\mathrm{p}$-values derived from t-test [11]. All analysis was performed using the R package (version 2.3.1). 


\section{Gene set enrichment analysis}

Gene set enrichment analysis (GSEA) was applied to assess the association between $\mathrm{pCR}, \mathrm{RD}$, and 852 distinct a priori defined gene sets. The goal of the GSEA is to determine whether members of a particular gene set (i.e. a list of 15-500 probe sets that correspond to genes that define a biological pathway) tend to occur toward the top or the bottom of a rank ordered gene list including all gene expression measurements [5]. We ranked all probe sets based on their correlation with pCR. Three groups of gene sets were tested, the first included 319 distinct gene sets corresponding to probes associated with 295 different cryptogenic bands on 24 chromosomes, the second included 522 different gene sets corresponding to genes involved in various metabolic and signaling pathways. A detailed description of these gene sets and how they were assembled was presented by Subramanian et al [5]. The third group contained 11 gene sets of various oncogenic or drug resistance related pathways. These included 5 distinct oncogenic pathways that were defined as genes overexpressed in normal human mammary epithelial cells transfected with Myc, Ras, E2F3, $\beta$-catenin and Src oncogenes, respectively and were described by Bild et. al [12]. One mutated p53-associated gene set that was defined as genes overexpressed in human breast cancer with known p53 mutation and was previously described by Miller et al [13]. It has been suggested that this gene expression signature can distinguish cancers with wild type and mutant p53 and it may outperform direct p53 gene sequencing as a predictor of prognosis and therapeutic response. We also assessed one ER-associated gene set that contained genes that were most highly coexpressed with the ER gene in human breast cancer microarray data developed by Symmans et al [14]. This gene set did not include the ER gene itself. We also examined the genomic grade index (GGI) that represents genes that are differentially expressed between low grade and high grade human breast cancers and were identified by Sotiriou et al [15]. One prognostic signature that was derived by comparing gene expression profiles of tumors that recurred with those that did not was also tested. This prognostic signature was first reported by Wang et al [16]. A proliferation signature set reported by Whitfield et al that includes genes involved in cell proliferation was also examined [17]. Finally, we also assessed an ATP-binding cassette transporter $(\mathrm{ABC})$ gene set that included genes involved in drug transport and was previously shown to predict chemotherapy response in cell lines by Szakacs et al [18].

Gene annotations were based on UniGene Build 185 that was used to match the genes in each of the above publication to probe sets on Affymetrix U133 A gene Chips. The gene sets are listed in Table 2 and a
Table 2 Gene sets used in this analysis

\begin{tabular}{|c|c|c|}
\hline Functional Pathway & $\begin{array}{l}\text { Number of } \\
\text { probe sets } \\
\text { (corresponding \# } \\
\text { of known genes) }\end{array}$ & Reference \\
\hline $\begin{array}{l}\text { Cytogenetic sets } \\
(n=319 \text { sets })\end{array}$ & 15-500 (variable) & Subramanian et al. [5] \\
\hline $\begin{array}{l}\text { Functional sets } \\
(n=522 \text { sets })\end{array}$ & 15-500 (variable) & Subramanian et al. [5] \\
\hline $\begin{array}{l}\text { Oncogenic Pathways } \\
\text { (5 sets) }\end{array}$ & & Bild et al. [10] \\
\hline Myc & 164 (139) & \\
\hline Ras & $228(176)$ & \\
\hline E2F3 & $173(147)$ & \\
\hline$\beta$-catenin & $54(42)$ & \\
\hline Src & $46(44)$ & \\
\hline $\begin{array}{l}\text { Genomic Grade Index } \\
\text { (1 set) }\end{array}$ & $242(183)$ & Sotiriou et al. [13] \\
\hline $\begin{array}{l}\text { 76-gene prognostic } \\
\text { signature ( } 1 \text { set) }\end{array}$ & $76(76)$ & Wang et al. [14] \\
\hline $\begin{array}{l}\text { Proliferation signature } \\
\text { (1 set) }\end{array}$ & $74(44)$ & Whitfield et al. [15] \\
\hline $\begin{array}{l}\text { ABC transporter gene set } \\
\text { (1 set) }\end{array}$ & $61(47)$ & Szakacs et al. [16] \\
\hline $\begin{array}{l}\text { Mutant-p53 signature } \\
\text { (1 set) }\end{array}$ & $25(21)$ & Miller et al. [11] \\
\hline $\begin{array}{l}\text { ER-associated gene set } \\
(1 \text { set) }\end{array}$ & $200(187)$ & Symmans et al. [12] \\
\hline
\end{tabular}

complete list of all probe sets that comprise each of the 852 sets is provided in supplementary Table 1 (Additional file 1).

Gene set enrichment score was calculated as reported previously [5]. This score is a measure of the degree to which a gene set is overrepresented at the extremes of the entire ranked gene list. Significance was assessed by permuting class labels (i.e. response category) and calculating enrichment scores for the permuted data sets that yielded a null distribution. Nominal p-value for a score was derived from comparison with this null distribution. In order to adjust for multiple hypothesis testing, the false discovery rate (FDR) q-value was calculated for each gene set. The q-value could be considered as an FDR-adjusted p-value; however unlike p-values which express the probability of a false-positive result for a single test, the q-value gives an estimate of the proportion of false positives for a set of results [19]. Gene sets with FDR q-value $\leq 0.25$ were considered to be of interest which indicates that the result is likely to be valid 3 out of 4 times and represents a previously proposed cut off in the literature [5]. Gene set enrichment analysis was performed using $R$ package of GSEA (version 1.0) provided by the Broad Institute of Massachusetts Institute of Technology (Cambridge, MA). 


\section{Results}

\section{Differentially expressed genes}

Twenty-seven out of 51 patients (53\%) had pCR among the ER-negative tumors and 7 out of 82 (9\%) among the ER-positive tumors. The much lower response rate in ER-positive cancers is consistent with previous reports in the clinical literature [1-4]. First, we examined if we could identify differentially expressed genes between cases with $\mathrm{pCR}$ and RD using unequal variance $\mathrm{t}$-test. We performed this analysis separately for ER-negative and ER-positive cancers. Figure 1 shows results of the BUM analysis of the $p$ values from the t-test. In ERnegative cancers, the FDR rate associated with the lowest $p$ value ( $p \leq 0.00087)$ was $40 \%$. In ER-positive cancers, the FDR was close to $100 \%$ for all observed $p$ values. The scarcity of low p-values in the ER-positive group is due to the unbalanced sample size (i.e. few informative cases, $7 \mathrm{pCR}$ only) and suggests and underpowered analysis that violates t-test assumptions.

These observations indicate that in these 2 data sets, t-test cannot reliably identify differentially expressed genes. However, these results do not necessarily indicate that there are no real transcriptional differences between cases with pCR compared to RD when ER-positive and ER-negative cancers are analyzed separately. It is possible, that no individual gene meets the threshold for statistical significance after correcting for multiple hypotheses testing because the transcriptional differences are modest relative to the technical noise and biological variability that is present in the data. Analysis at the single gene level may also miss small but coordinated expression differences in a larger number of genes that could belong to important biological pathways. In some situations, small coordinated change in the expression of many genes that belong to a particular metabolic pathway can have robust functional consequences [20]. Such subtle gene expression differences would not be identified easily by pair-wise comparisons using t-statistics. Different analytical tools, including gene set enrichment analysis (GSEA) were developed to test for potentially relevant but small scale transcriptional differences in pre-defined sets of genes.

\section{Gene set enrichment analysis to identify pathways associated with complete response to preoperative chemotherapy}

We applied GSEA to the 51 ER-negative tumors. Only 3 gene sets out of the 853 were enriched with an FDR q $\leq$ 0.25 . These gene sets included; (i) the proliferation set $($ FDR $q=0.1, p=0.05)$ (Figure 2A and Supplementary Figure 1A in Additional file 2); (ii) the genomic grade index set (FDR q $=0.04, p=0.08$ ) (Figure $2 B$ and Supplementary Figure 1B in Additional file 2); and (iii) the E2F3 pathway gene set $(F D R q=0.2, \mathrm{p}=0.07$ ) (Figure $2 \mathrm{C}$ and

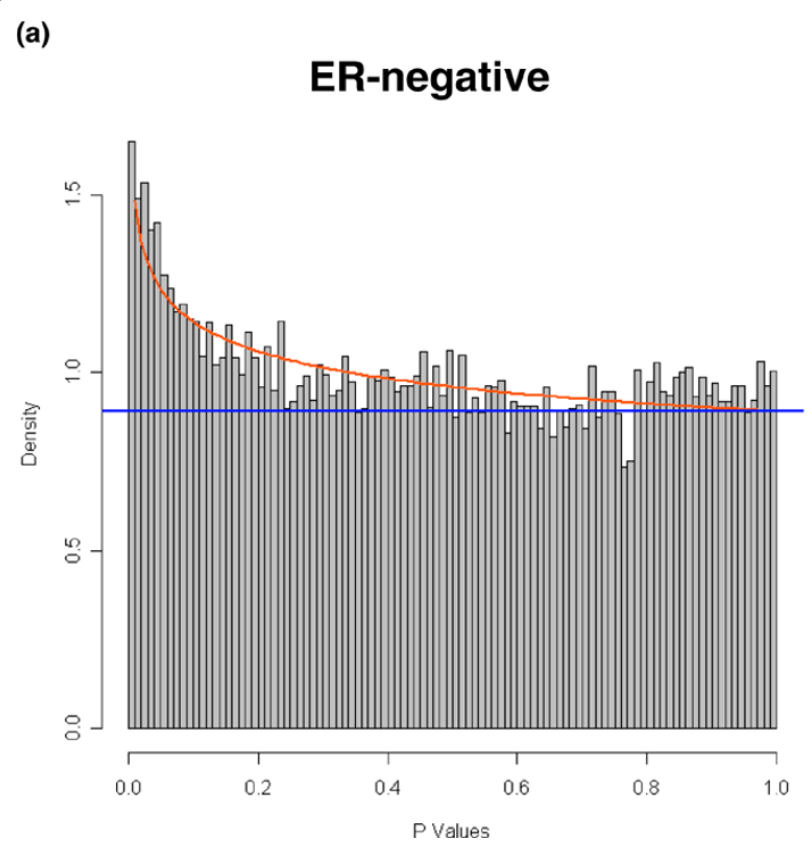

(b)

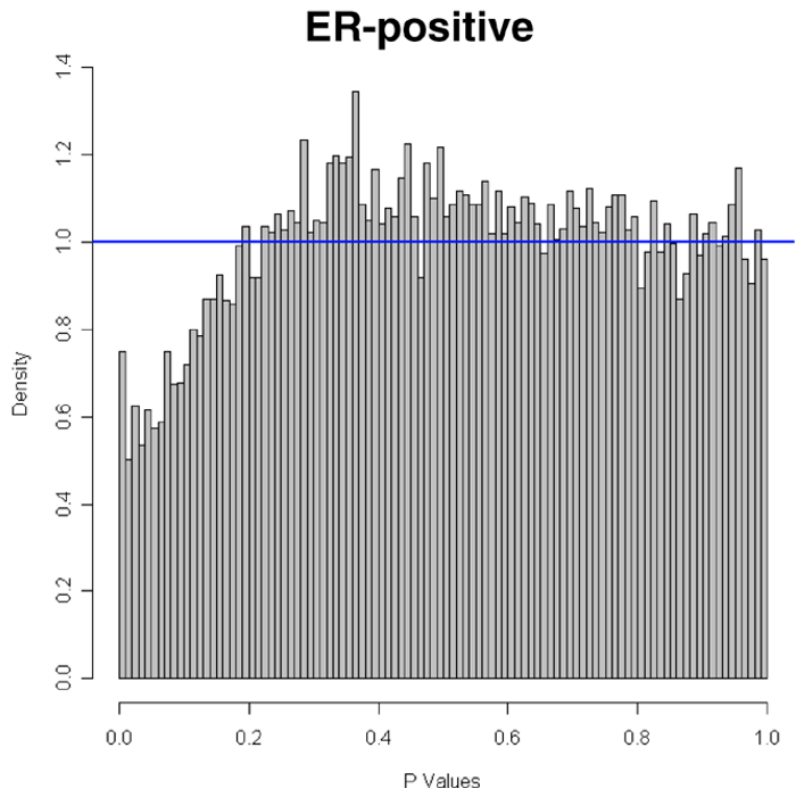

Figure 1 Distribution of $p$ values computed from unequal variance t-test in patients with ER-negative and ER-positive tumors, respectively. A, Gene expressions were compared between ER-negative tumors that had pathologic complete response and those that had a lesser response to preoperative chemotherapy. The resulting p-values for all comparisons were modeled as beta uniform mixture. The straight line indicates the contribution of the uniform component, and the curved line is the fitted beta-distribution from the observed values. Deviation above the straight line indicate $p$-values that may represent true discovery. B, Distribution of $p$ values in patients with ER-positive tumors. 


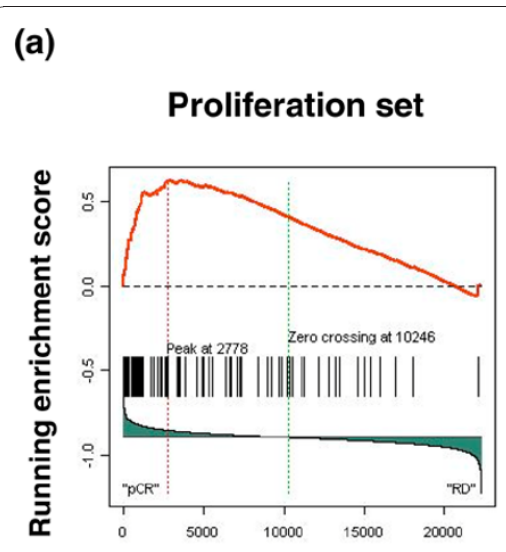

Rank in ordered dataset (b)

\section{Genomic grade index}

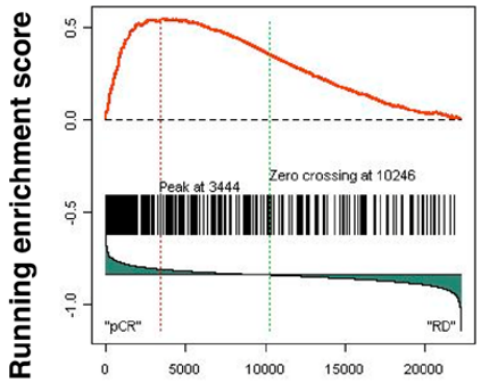

Rank in ordered dataset (c)

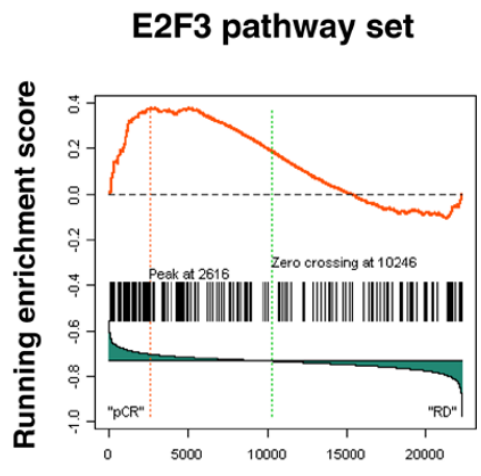

Rank in ordered dataset

Figure 2 Gene set enrichment results for ER-negative breast cancers. Running enrichment scores (RES) and the location of each probe set within the complete ranked ordered gene list for each gene set. The first dotted line indicates the position of the maximum RES, the second dotted line indicates the zero position of the ranking metric score. A, proliferation set (probe set $n=74$ ). B, genomic grade index (probe set $n=242$ ). C, E2F3 pathway (probe set $n=173$ ) ( $\mathrm{PCR}=$ pathologic complete response; $\mathrm{RD}=$ residual disease). Heat maps corresponding to these plots are provided on supplementary figure 1 in Additional file 2 .

Supplementary Figure $1 \mathrm{C}$ in Additional file 2). All of these were enriched in the group with pathologic complete response, while no gene set was enriched in the group with residual cancer.

We performed the same analysis on the 82 ER-positive cases. Two gene sets were enriched in the group with pathologic complete response including the proliferation set $($ FDR $q=0.001, p=0.002)$ (Figure 3A and Supplementary Figure 2A in Additional file 3 ) and the genomic grade index set (FDR q $=0.015, \mathrm{p}=0.01$ ) (Figure $3 \mathrm{~B}$ and Supplementary Figure 2B in Additional file 3). In the group with residual cancer, 2 other gene sets showed enrichment including the ER-associated gene list (FDR $\mathrm{q}=0.03, \mathrm{p}=0.04$ ) (Figure $3 \mathrm{C}$ and Supplementary Figure $2 \mathrm{C}$ in Additional file 3 ) and the mutant p53 gene signature (FDR q $=0.0019, \mathrm{p}=0.07$ ) (Figure 3D and Supplementary Figure 2 D in Additional file 3). The complete list of probes and genes included in the five enriched gene sets is presented in supplementary Table 2 (Additional file 4).

These results indicate that higher expression of proliferation-related genes characterized cancers with pCR among both ER-negative and ER-positive cancers. There are several single gene markers of proliferative activity; the one that is most commonly used in the clinic is Ki67 (which was also included in the proliferation gene set). We therefore examined if measuring Ki67 (MIK67) mRNA expression alone is sufficient to separate cases with pCR from those with residual disease after chemotherapy. Ki67 expression is measured by 2 distinct Affymetrix probe sets "212021_s_at" and "212023_s_at". In ER-negative cancers, neither of these probe sets were significantly differentially expressed according to pathologic response to chemotherapy (unequal variance t-test, $\mathrm{p}=0.97$ and 0.92, respectively). In ER-positive cancers, one of the 2 probe sets ("212023_s_at") showed borderline significant over-expression in the pathologic complete response group, $\mathrm{p}=0.06$.

Also, since the ER-associated gene set was enriched in ER-positive breast cancers with residual disease after chemotherapy, we examined if quantitative assessment of the ER (ESR1) mRNA alone could provide the same information [21]. There was no statistically significant difference in ER mRNA expression levels (probe set "205225_at") between cases with complete response and those with residual cancer after chemotherapy (unequal variance $\mathrm{t}$-test, $\mathrm{p}=0.09$ ) among the ER-positive tumors.

\section{Discussion}

In the present study, we examined if we could find individual genes or gene sets that are significantly associated with extreme chemotherapy sensitivity in ER-positive and ER-negative breast cancers, respectively. These two major types of breast cancers differ in the expression of thousands of genes $[6,7,22]$. They also have substantially different sensitivity to cytotoxic treatment; ER-positive cancers are generally less sensitive to chemotherapy than ER-negative tumors [2]. Therefore, when these cancers are analyzed together, sensitivity markers tend to identify ER-negative tumors and are often dominated by genes that reflect the ERstatus of the tumor [4]. In order to identify markers of response that are independent of ER-status we analyzed these 2 groups of breast cancers separately. To our surprise, the commonly used approach which performs gene wise comparison between responders and 

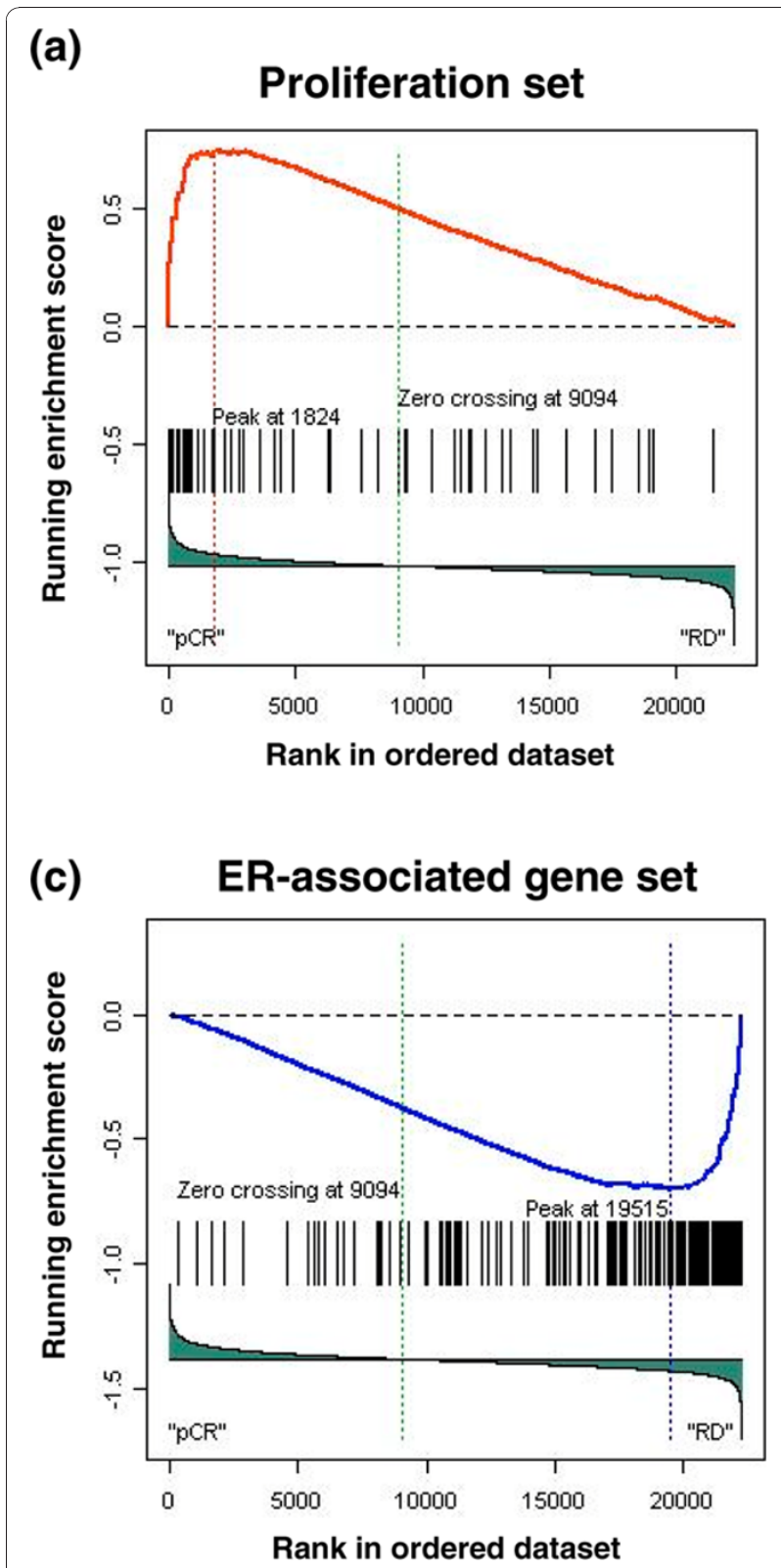

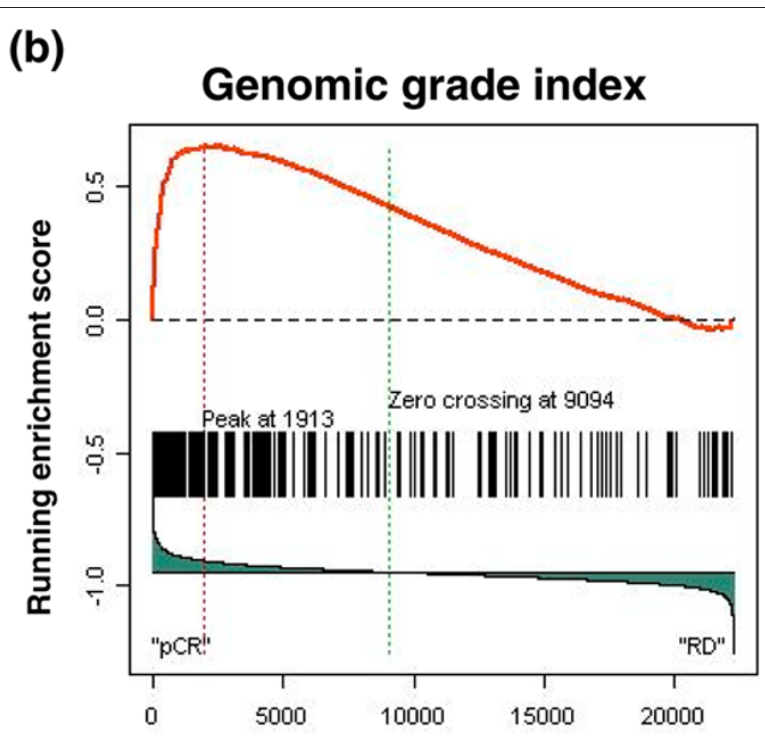

Rank in ordered dataset
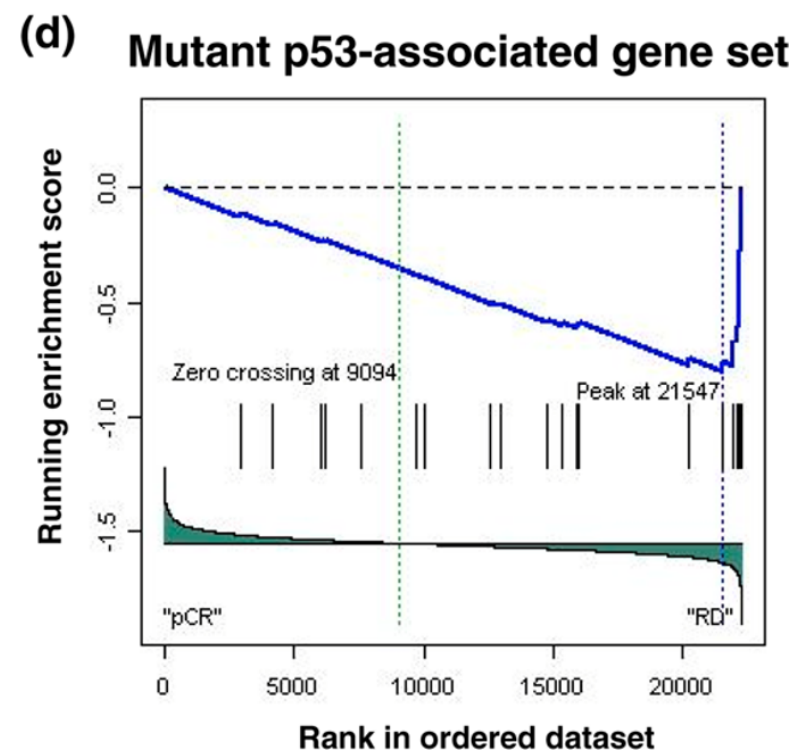

Figure 3 Gene set enrichment results for ER-positive breast cancers. Results are presented as in figure 2. A, proliferation set. B, genomic grade index: $\mathbf{C}$, ER-associated genes (probe set $n=201$ ). $\mathbf{D}$, mutant p53 gene signature (probe set $n=25$ ). Heat maps corresponding to these plots are provided on supplementary figure 2 in Additional file 3.

non-responders failed to identify any genes that could be declared differentially expressed with statistical confidence. The estimated false discovery rate was $>40 \%$ among the top differentially expressed genes in ERnegative cancers and the FDR was even higher among ER-positive cancers. These findings are in contrast with the results that can be obtained when the entire patient cohort is analyzed together. When we searched for differentially expressed genes including both ERnegative and -positive cases, we could identify over 400 genes with an FDR $\leq 1 \%$ [4].
We next examined if coordinated but relatively small scale differences in the expression of sets of genes that belong to functional pathways are associated with response. Such small scale differences at the individual gene level may not be readily identified by $t$-statistics but gene set enrichment analysis may be able to detect these. Two gene sets emerged as strongly enriched in cancers with pCR to chemotherapy in both ER-positive and ER-negative cancers. These included 44 genes (corresponding to 74 probe sets) involved in cell proliferation and 183 genes (corresponding to 242 probe sets) 
that distinguish histologically high grade cancers from low grade tumors. These observations are consistent with the literature that suggests that highly proliferative cancers are more sensitive to cytotoxic treatment in general [23]. However, there is no consensus how to best measure proliferative activity [24]. To underscore the power of gene set analysis, we noted that the proliferation signature as a whole was significantly overrepresented in highly chemotherapy sensitive tumors. However, a commonly used proliferation marker Ki67 that was included in the signature showed no significant over-expression when tested alone. It is also well documented in the clinical literature that high histological grade is associated with better response to preoperative chemotherapy [25]. It was reassuring to observe that the same association holds up for the genomic grade index too.

We also made 3 novel observations. Our results indicate that the expression of genes involved in the E2F3 pathway may be associated with high degree of chemotherapy sensitivity in ER-negative cancers. Considering that the E2F3 family of transcription factors plays a critical role in regulating cell cycle progression this association is not surprising [26]. Nevertheless, no previous reports linked E2F3 activity to chemotherapy response. It is also intriguing that no association between E2F3 pathway and pCR was seen in ER-positive cancers. We also observed higher expression of mutant-p53 associated genes in relatively chemotherapy resistant ER-positive breast cancers. A similar association was not seen among ER-negative cancers, which suggests that p53 dysregulation may have different consequences on chemotherapy sensitivity depending on the hormone receptor status of the cancer. This may partly explain the conflicting results about the role of p53 mutation in chemotherapy response in the literature. Some studies suggested that functional p53 defects predict for increased sensitivity to anthracycline chemotherapy [27]. Others reported that p53 mutations are associated with resistance to anthracyclines $[28,29]$. It has also been shown that breast cancer cell lines exhibit different transcriptional response to chemotherapy in vitro depending on their hormone receptor status and molecular class. For example, the expression of p21, a p53-regulated protein, was highly induced in ER-positive cells, but only weakly in ERnegative breast cancer cell lines in response to anthracycline exposure [30]. This suggests that p53-mediated apoptosis may be more important in ER-positive (luminal) than in ER-negative (basal-like) cells. Future biomarker studies will need to consider the possibility that the predictive value of a biomarker may depend on the molecular subtype of the cancer [31].
We also observed that those ER-positive cancers that had low expression of ER-associated genes were more sensitive to chemotherapy. This was independent of the actual level of ER expression and indicates that some ER-positive breast cancers do not posses the full transcriptional signature of ER-activity. These cancers showed increased chemotherapy sensitivity.

Our study has limitations. All patients received combination chemotherapy that represents the current standard of care for this patient population. This makes our observations more relevant for clinical practice but at the same time limits our ability to decipher drug-specific response pathways. This could have biased our results towards detecting "generic" drug sensitivity pathways such as proliferation. The gene sets that we tested were assembled from the published literature and often contain overlapping genes represented in multiple gene sets. Our current knowledge of biology is incomplete and does not allow defining precisely all the genes that contribute to a given biological process or represent a unique molecular pathway. This may explain why the majority of the 852 gene sets that we examined including numerous apoptosis and signaling pathways did not show enrichment by chemotherapy response. It is also important to consider that GSEA is a method to demonstrate that the expression of a given gene set is overrepresented in a the top or bottom of particular gene lists ranked by correlation with clinical outcome. However, this method cannot be used to predict response in a new case. How to translate GSEA results into a prospective, single sample response predictor remains an unsolved bioinformatics challenge.

\section{Conclusion}

We found that it is difficult to identify individual genes associated with chemotherapy response with statistical confidence when ER-negative and -positive breast cancers are analyzed separately. In contrast, GSEA revealed several biological pathways that were associated with response. These included proliferation related genes and the genomic grade index that were enriched in cancers with high sensitivity to chemotherapy regardless of ER status. Genes included in the E2F3 pathway were also enriched in ER-negative and highly chemotherapy sensitive cancers. On the other hand, a mutant p53 gene expression signature and a set of highly ER-associated genes were enriched in ER-positive and chemotherapy resistant cancers. These results suggest that proliferative activity confers increased sensitivity to chemotherapy in breast cancer in general, whereas other biological pathways such p53 mutation, E2F3 activation may be more selective and influence chemotherapy sensitivity only in particular molecular subtypes of breast cancer. 


\section{Additional material}

Additional file 1: Supplementary Table 1 includes a complete list of all probe sets that comprise each of the 852 gene sets.

Additional file 2: Supplementary Figure 1 presents the same plots as Figure 1 for ER-negative cancers but also includes heat maps of the proliferation set (FDR $q=0.1, p=0.05$ ) (Supplementary Figure $1 \mathrm{~A}$ ); the genomic grade index set (FDR $q=0.04, p=0.08$ ) (Supplementary Figure $1 \mathrm{~B})$; and the E2F3 pathway gene set (FDR $q=0.2, p=0.07$ )

(Supplementary Figure $1 C$ ). All of these were enriched in the group with pathologic complete response, while no gene set was enriched in the group with residual cancer.

Additional file 3: Supplementary Figure $\mathbf{2}$ presents the same plots as Figure 3 for ER-positive cancers but also includes heat maps of the proliferation set (FDR $q=0.001, p=0.002$ ) (Supplementary Figure 2A) and the genomic grade index set (FDR $q=0.015, p=0.01$ ) (Supplementary Figure 2B). In the group with residual cancer, 2 other gene sets showed enrichment including the ER-associated gene list (FDR $q=0.03, p=0.04$ ) Supplementary Figure $2 C$ ) and the mutant p53 gene signature (FDR $q=0.0019, p=0.07$ ) (Supplementary Figure 2D).

Additional file 4: Supplementary Table 2 includes the complete list of probes and genes included in the five enriched gene sets.

\section{Abbreviations}

ABC: ATP-binding cassette transporter; BUM: beta-uniform mixture; ER: estrogen receptor; FDR: false discovery rate; GGI: genomic grade index; GSEA: gene set enrichment analysis; pCR: pathologic complete response; RD: residual disease.

\section{Acknowledgements}

Supported by grants to L.P. from the NCI (RO1-CA106290), the Breast Cancer Research Foundation, and the Goodwin Foundation and to G.N.H. by the Nellie B. Connally Breast Cancer Research Fund. T.A. is a visiting professor of the Hungarian American Enterprise Scholarship Fund (HAESF). F.A is supported by Fondation de France, Fondation Lilly and a career development award from the American Society of Clinical Oncology. C.L. is supported by the Deutsche Forschungsgemeinschaft.

\section{Author details}

${ }^{1}$ Department of Breast Medical Oncology, The University of Texas M. D. Anderson Cancer Center, Houston, TX. ${ }^{2}$ Departments of Bioinformatics and Computation Biology, The University of Texas M. D. Anderson Cancer Center, Houston, TX. ${ }^{3}$ Department of Pathology of The University of Texas M. D. Anderson Cancer Center, Houston, TX. ${ }^{4}$ Department of Molecular Diagnostics, National Medical Center, Budapest, Hungary. ${ }^{5}$ Unit UPRES03535, Institut Gustave Roussy, Villejuif, France. ${ }^{6} J u l e s$ Bordet Institut, Brussels, Belgium.

\section{Authors' contributions}

AT, JW, FA, CL and LP developed the idea of the study, assembled the molecular and clinical data and performed the evaluation of results, preparation of figures, drafting and finalyzing the manuscript. JW and KY performed the statistical analyses. WFS generated the gene expression data and WFS, CS, and GNH helped draft and revise the manuscript. All authors read and approved the final manuscript.

\section{Competing interests}

The authors declare that they have no competing interests.

Published: 29 April 2008

\section{References}

1. Kaufmann M, von Minckwitz G, Smith R, Valero V, Gianni L, Eiermann W, Howell A, Costa SD, Beuzeboc P, Untch M, Blohmer JU, Sinn HP, Sittek R, Souchon R, Tulusan AH, Volm T, Senn HJ: International expert panel on the use of primary (preoperative) systemic treatment of operable breast cancer: review and recommendations. J Clin Onc 2003, 21:2600-8.

2. Mazouni C, Kau SW, Frye D, Andre F, Buchholz TA, Symmans WF, Anderson K, Hess KR, Gonzalez-Angulo AM, Hortobagyi GN, Buzdar AU, Pusztai L: Inclusion of taxanes, particularly weekly paclitaxel, in preoperative chemotherapy improves pathologic complete response rate in ER-positive breast cancers. Annals of Oncology 2007, 18:878-80.

3. Kuerer HM, Newman LA, Smith TL, Ames FC, Hunt KK, Dhingra K, Theriault RL, Singh G, Binkley SM, Sneige N, Buchholz TA, Ross MI, McNeese MD, Buzdar AU, Hortobagyi GN, Singletary SE: Clinical course of breast cancer patients with complete pathologic primary tumor and axillary lymph node response to doxorubicin-based neoadjuvant chemotherapy. J Clin Oncol 1999, 17:460-9.

4. Hess KR, Anderson K, Symmans WF, Valero V, Ibrahim N, Mejia JA, Booser D, Theriault RL, Buzdar AU, Dempsey PJ, Rouzier R, Sneige N, Ross JS, Vidaurre T, Gómez HL, Hortobagyi GN, Pusztai L: Pharmacogenomic predictor of sensitivity to preoperative chemotherapy with paclitaxel and fluorouracil, doxorubicin, and cyclophosphamide in breast cancer. J Clin Oncol 2006, 24:4236-44.

5. Subramanian A, Tamayo P, Mootha VK, Mukherjee S, Ebert BL, Gillette MA, Paulovich A, Pomeroy SL, Golub TR, Lander ES, Mesirov JP: Gene set enrichment analysis: a knowledge-based approach for interpreting genome-wide expression profiles. Proc Natl Acad Sci USA 2005, 102:15545-50.

6. Gruvberger S, Ringner M, Chen Y, Panavally S, Saal LH, Borg A, Fernö M, Peterson C, Meltzer PS: Estrogen receptor status in breast cancer is associated with remarkably distinct gene expression patterns. Cancer Research 2001, 61:5979-84.

7. Perou CM, Sorlie T, Eisen MB, van de Rijn M, Jeffrey SS, Rees CA, Pollack JR, Ross DT, Johnsen $H$, Akslen LA, Fluge O, Pergamenschikov A, Williams C, Zhu SX, Lønning PE, Børresen-Dale AL, Brown PO, Botstein D: Molecular portraits of human breast tumours. Nature 2000, 406:747-52.

8. Symmans WF, Ayers M, Clark EA, Stec J, Hess KR, Sneige N, Buchholz TA, Krishnamurthy S, Ibrahim NK, Buzdar AU, Theriault RL, Rosales MF, Thomas ES, Gwyn KM, Green MC, Syed AR, Hortobagyi GN, Pusztai L: Total RNA yield and microarray gene expression profiles from fine needle aspiration and core needle biopsy samples of breast cancer. Cancer 2003, 97:2960-2971.

9. [http://dChip.org].

10. [http://bioinformatics.mdanderson.org/pubdata.html].

11. Pounds S, Morris SW: Estimating the occurrence of false positive and false negatives in microarray studies by approximating and partitioning the empirical distribution of p-values. Bioinformatics 2003, 19:1236-42.

12. Bild AH, Yao G, Chang JT, Wang Q, Potti A, Chasse D, Joshi MB, Harpole D, Lancaster JM, Berchuck A, Olson JA Jr, Marks JR, Dressman HK, West M, Nevins JR: Oncogenic pathway signatures in human cancers as a guide to targeted therapies. Nature 2006, 439:353-7.

13. Miller LD, Smeds J, George J, Vega VB, Vergara L, Ploner A, Pawitan Y, Hall P, Klaar S, Liu ET, Bergh J: An expression signature for p53 status in human breast cancer predicts mutation status, transcriptional effects, and patient survival. Proc Natl Acad Sci USA 2005, 102:13550-5.

14. Symmans WF, Sotirion C, Anderson SK, et al: Measurements of estrogen receptor and reporter genes from micro arrays determine receptor status and time to recurrence following adjuvant tamoxifen therapy. Breast Cancer Res Treat 2005, 94:S32, (Abst \#308).

15. Sotiriou C, Wirapati P, Loi S, Harris A, Fox S, Smeds J, Nordgren H, Farmer P, Praz V, Haibe-Kains B, Desmedt C, Larsimont D, Cardoso F, Peterse H, Nuyten D, Buyse M, Van de Vijver MJ, Bergh J, Piccart M, Delorenzi M: Gene expression profiling in breast cancer: understanding the molecular basis of histologic grade to improve prognosis. J Natl Cancer Inst 2006, 98:262-72.

16. Wang Y, Klijn JG, Zhang Y, Sieuwerts AM, Look MP, Yang F, Talantov D, Timmermans M, Meijer-van Gelder ME, Yu J, Jatkoe T, Berns EM, Atkins D, Foekens JA: Gene-expression profiles to predict distant metastasis of lymph-node-negative primary breast cancer. Lancet 2005, 365:671-9.

17. Whitfield ML, George LK, Grant GD, Perou CM: Common markers of proliferation. Nat Rev Cancer 2006, 6:99-106.

18. Szakacs G, Annereau JP, Lababidi S, Shankavaram U, Arciello A, Bussey K, Reinhold W, Guo Y, Kruh GD, Reimers M, Weinstein JN, Gottesman MM: Predicting drug sensitivity and resistance: profiling $A B C$ transporter genes in cancer cells. Cancer Cell 2004, 6:129-37. 
19. Storey JD: A direct approach to false discovery rates. J Roy Stat Soc Ser B Meth 2002, 64:479-98.

20. Mootha VK, Lindgren CM, Eriksson KF, Subramanian A, Sihag S, Lehar J, Puigserver P, Carlsson E, Ridderstråle M, Laurila E, Houstis N, Daly MJ, Patterson N, Mesirov JP, Golub TR, Tamayo P, Spiegelman B, Lander ES, Hirschhorn JN, Altshuler D, Groop LC: PGC-1alpha-responsive genes involved in oxidative phosphorylation are coordinately down-regulated in human diabetes. Nature Genetics 2003, 34:267-73.

21. Gong Y, Yan K, Lin F, Anderson K, Sotiriou C, Andre F, Holmes FA, Valero V, Booser D, Pippen JE Jr, Vukelja S, Gomez H, Mejia J, Barajas LJ, Hess KR, Sneige N, Hortobagyi GN, Pusztai L, Symmans WF: Determination of oestrogen-receptor status and HER-2 status of breast carcinoma: a geneexpression profiling study. Lancet Oncol 2007, 8:203-211.

22. Pusztai L, Ayers M, Stec J, Clark E, Hess K, Stivers D, Damokosh A, Sneige N, Buchholz TA, Esteva FJ, Arun B, Cristofanilli M, Booser D, Rosales M, Valero V, Adams C, Hortobagyi GN, Symmans WF: Gene expression profiles obtained from single passage fine needle aspirations (FNA) of breast cancer reliably identify prognostic/predictive markers such as estrogen (ER) and HER-2 receptor status and reveal large scale molecular differences between ER-negative and ER-positive tumors. Clinical Cancer Res 2003, 9:2406-2415.

23. Vincent-Salomon A, Rousseau A, Jouve M, Beuzeboc P, Sigal-Zafrani B, Fréneaux P, Rosty C, Nos C, Campana F, Klijanienko J, Al Ghuzlan A, SastreGarau X, Breast Cancer Study Group: Proliferation markers predictive of the pathological response and disease outcome of patients with breast carcinomas treated by anthracycline-based preoperative chemotherapy. Eur J Cancer 2004, 40:1502-8.

24. Ross JS, Symmans WF, Pusztai L, Hortobagyi GN: Breast cancer biomarkers. Adv Clin Chem 2006, 40:99-125

25. Ellis P, Smith I, Ashley S, Walsh G, Ebbs S, Baum M, Sacks N, McKinna J: Clinical prognostic and predictive factors for primary chemotherapy in operable breast cancer. J Clin Oncol 1998, 16:107-14.

26. DeGregori J: The genetics of the E2F family of transcription factors: shared functions and unique roles. Biochim Biophys Acta 2002, 1602:131-50.

27. Bertheau P, Plassa F, Espie M, Turpin E, de Roquancourt A, Marty M, Lerebours F, Beuzard Y, Janin A, de Thé H: Effect of mutated TP53 on response of advanced breast cancers to high-dose chemotherapy. Lancet 2002, 360:852-4

28. Aas T, Borresen AL, Geisler S, Smith-Sørensen B, Johnsen H, Varhaug JE, Akslen LA, Lønning PE: Specific P53 mutations are associated with de novo resistance to doxorubicin in breast cancer patients. Nat Med 1996, 2:811-4.

29. Di Leo A, Tanner A, Desmedt C, Paesmans M, Cardoso F, Durbecq V, Chan S, Perren T, Aapro M, Sotiriou C, Piccart MJ, Larsimont D, Isola J, TAX 303 translational study team: P53 gene mutations as a predictive marker in a population of advanced breast cancer patients randomly treated with doxorubicin or docetaxel in the context of a Phase III clinical trial. Ann Oncol 2007, 18:997-1003.

30. Troester MA, Hoadley KA, Sorlie T, Herbert BS, Børresen-Dale AL, Lønning PE, Shay JW, Kaufmann WK, Perou CM: Cell-type-specific responses to chemotherapeutics in breast cancer. Cancer Res 2004, 64:4218-4226.

31. Andre F, Mazouni C, Hortobagyi GN, Pusztai L: DNA arrays as predictors of efficacy of adjuvant/neoadjuvant chemotherapy in breast cancer patients: Current data and issues on study design. Biochim Biophys Acta 2006, 1766:197-204.

doi: $10.1186 / \mathrm{bcr} 2088$

Cite this article as: Tordai et al: Evaluation of biological pathways involved in chemotherapy response in breast cancer. Breast Cancer Research 2008 10:R37.

\section{Submit your next manuscript to BioMed Central and take full advantage of:}

- Convenient online submission

- Thorough peer review

- No space constraints or color figure charges

- Immediate publication on acceptance

- Inclusion in PubMed, CAS, Scopus and Google Scholar

- Research which is freely available for redistribution

Submit your manuscript at www.biomedcentral.com/submit
Biomed Central 